\title{
On a form of the Erdös-Turán inequality
}

by

Jeffrey J. Holt (Houghton, Mich.)

1. Introduction. Let $\mathcal{P}=\left\{x_{1}, \ldots, x_{N}\right\}$ be a set of points in $\mathbb{R}$, and define the $\mathbb{Z}$-periodic set

$$
\mathcal{P}^{*}=\{x+m: x \in \mathcal{P}, m \in \mathbb{Z}\} .
$$

The discrepancy $D(\mathcal{P})$ gives a measure of how evenly (or unevenly) distributed $\mathcal{P}$ is in $\mathbb{R} / \mathbb{Z}$. There are a number of ways to define the discrepancy (see, for instance, $[2,8,13]$ ); a common form is as follows: Let $s, t$ be real numbers which satisfy $s<t<s+1$, and let $\chi_{s, t}(x)$ denote the characteristic function of the interval $[s, t]$. Then we define

$$
D(\mathcal{P})=\sup _{s<t<s+1}\left|\sum_{x \in \mathcal{P}^{*}} \chi_{s, t}(x)-N(t-s)\right| .
$$

In 1948, P. Erdős and P. Turán [4] established a quantitative connection between $D(\mathcal{P})$ and the exponential sums

$$
\left|\sum_{n=1}^{N} e\left(m x_{n}\right)\right|
$$

where $m$ is a nonzero integer and $e(\theta)=e^{2 \pi i \theta}$. Specifically, they showed that there exist absolute constants $C_{1}$ and $C_{2}$ such that

$$
D(\mathcal{P}) \leq C_{1} N M^{-1}+C_{2} \sum_{m=1}^{M} m^{-1}\left|\sum_{n=1}^{N} e\left(m x_{n}\right)\right|
$$

holds for all integers $M \geq 1$. Explicit values for $C_{1}$ and $C_{2}$ are given in ([8], pp. 112-114) and ([12], Theorem 20).

The notion of the discrepancy of a point set has been generalized to a wide variety of settings. Bounds in the style of (1.2) have been given in several cases (see $[3,5,11]$ ), and are typically referred to as "Erdős-Turán" inequalities. Here we establish such an inequality for points distributed on the unit torus $\mathbb{R}^{k} / \mathbb{Z}^{k}$, where $k \geq 2$. In a manner analogous to the onedimensional case, we let $\mathcal{P}=\left\{\mathbf{x}_{1}, \ldots, \mathbf{x}_{N}\right\}$ be a set of points in $\mathbb{R}^{k}$ and then 
define

$$
\mathcal{P}^{*}=\left\{\mathbf{x}+\mathbf{m}: \mathbf{x} \in \mathcal{P}, \mathbf{m} \in \mathbb{Z}^{k}\right\} .
$$

For $r>0$ and $\mathbf{c} \in \mathbb{R}$, let $B_{k}(r, \mathbf{c})$ denote the closed ball of radius $r$ centered at $\mathbf{c}$ given by

$$
B_{k}(r, \mathbf{c})=\left\{\mathbf{x} \in \mathbb{R}^{k}:|\mathbf{x}-\mathbf{c}| \leq r\right\},
$$

where $|\cdot|$ denotes the usual Euclidean metric on $\mathbb{R}^{k}$. For each such $r$ and $\mathbf{c}$, define

$$
\Delta\left[\mathcal{P} ; B_{k}(r, \mathbf{c})\right]=Z\left[\mathcal{P}^{*} ; B_{k}(r, \mathbf{c})\right]-N \mu\left(B_{k}(r, \mathbf{c})\right),
$$

where $Z[Q ; A]$ denotes the number of points of a discrete set $Q \subset \mathbb{R}^{k}$ which fall in a compact set $A \subset \mathbb{R}^{k}$, and $\mu$ is the usual Euclidean volume. For each $r>0$ we then define the discrepancy $D_{r}(\mathcal{P})$ by

$$
D_{r}(\mathcal{P})=\sup _{\mathbf{c} \in \mathbb{R}^{k}}\left|\Delta\left[\mathcal{P} ; B_{k}(r, \mathbf{c})\right]\right| .
$$

By applying an observation of H. L. Montgomery (see [1], Section 2.3) together with functions constructed by J. Vaaler and the author [7], we establish the following bound:

Theorem 1. Let $r>0$ and $\mathcal{P}=\left\{\mathbf{x}_{1}, \ldots, \mathbf{x}_{N}\right\}$ be a subset of $\mathbb{R}^{k}$. Then

$$
D_{r}(\mathcal{P})
$$

$$
\leq N A_{k}(r, s)+\sum_{|\mathbf{m}|<s}^{\prime}\left\{A_{k}(r, s)+(r /|\mathbf{m}|)^{k / 2}\left|J_{k / 2}(2 \pi r|\mathbf{m}|)\right|\right\}\left|\sum_{n=1}^{N} e\left(\mathbf{m} \cdot \mathbf{x}_{n}\right)\right|
$$

for all $s>0$, where

$$
\begin{aligned}
A_{k}(r, s)= & \omega_{k} s^{-1} r^{k-1}\left\{\pi r s\left(J_{(k-2) / 2}(\pi r s)^{2}+J_{k / 2}(\pi r s)^{2}\right)\right. \\
& \left.-(k-1) J_{(k-2) / 2}(\pi r s) J_{k / 2}(\pi r s)\right\}^{-1},
\end{aligned}
$$

$J_{\nu}(x)$ is the $\nu$-th order Bessel function and $\omega_{k}=4 \pi^{(k-2) / 2} \Gamma(k / 2)^{-1}$.

In 1969, W. Schmidt [9] showed that the discrepancy cannot be uniformly small. Suppose that $\varepsilon>0$ and that $\delta$ satisfies $N \delta^{k} \geq 1$. Schmidt proved that there exists a ball $B_{k}(r, \mathbf{c})$ with $r \leq \delta$ such that

$$
\left|\Delta\left[\mathcal{P} ; B_{k}(r, \mathbf{c})\right]\right|>c_{1}(k, \varepsilon)\left(N \delta^{k}\right)^{(k-1) / 2 k-\varepsilon} .
$$

On the other hand, J. Beck ([2], Theorem 14) has shown that there exists an infinite sequence $\mathbf{x}_{1}, \mathbf{x}_{2}, \ldots$ such that for all $N \geq 2$ and any ball $B_{k}(r, \mathbf{c})$ with $r \leq 1$ and $N r^{k} \geq 1$, we have

$$
\left|\Delta\left[\left\{\mathbf{x}_{1}, \ldots, \mathbf{x}_{N}\right\} ; B_{k}(r, \mathbf{c})\right]\right| \leq c_{2}(k)\left(N r^{k}\right)^{(k-1) / 2 k}(\log N)^{3 / 2} .
$$

In view of Schmidt's lower bound, we see that (1.6) must be close to best possible. Applying Theorem 1 and a simple averaging procedure, we may establish the existence of a set of $p$ points in $\mathbb{R}^{k}$ (here $p$ is a prime) which 
has modest discrepancy. (A similar application is given in [8], pp. 154-157.) Although our result is not as sharp as Beck's, our proof is much simpler and we do not have the requirement that $r \leq 1$. For $\mathbf{h} \in \mathbb{Z}^{k}$, let $\mathcal{P}_{\mathbf{h}}$ be the collection of $p$ points of the form $(n / p) \mathbf{h}, n=1, \ldots, p$.

TheOREM 2. Let $p$ be a prime number, and suppose that $r \geq p^{-1 / k}$. Then there exists a lattice point $\mathbf{h} \in \mathbb{Z}^{k}$ such that $|\mathbf{h}|<p$ and

$$
D_{r}\left(\mathcal{P}_{\mathbf{h}}\right) \leq c_{3}(k)\left(p r^{k}\right)^{(k-1) / k} .
$$

Notation. We use the definition for Fourier transforms and series of Stein and Weiss [10]. For $\mathbf{x} \in \mathbb{R}^{k}$ and $r>0, \chi_{r}(\mathbf{x})$ denotes the characteristic function of $B_{k}(r, \mathbf{0})$. To simplify expressions, we adopt the convention that $\mathbf{m}$ appearing in a sum will always be a point in $\mathbb{Z}^{k}$. Finally, $\sum^{\prime}$ means that the term in the sum corresponding to $\mathbf{m}=\mathbf{0}$ is omitted.

\section{Proof of theorems}

Pro of of Theorem 1. We require two auxiliary functions. Combining the results in ([7], Theorem 3) and a $k$-dimensional form of the PaleyWiener theorem ([10], Chapter III, Theorem 4.9), we see that for $r>0$ and $s>0$ there exist functions $\mathcal{F}_{k}(\mathbf{x} ; r, s)$ and $\mathcal{G}_{k}(\mathbf{x} ; r, s)$ that satisfy

$$
\begin{gathered}
\mathcal{F}_{k}(\mathbf{x} ; r, s) \leq \chi_{r}(\mathbf{x}) \leq \mathcal{G}_{k}(\mathbf{x} ; r, s) \quad \text { for all } \mathbf{x} \in \mathbb{R}^{k}, \\
\widehat{\mathcal{F}}_{k}(\mathbf{t} ; r, s)=\widehat{\mathcal{G}}_{k}(\mathbf{t} ; r, s)=0 \quad \text { for all }|\mathbf{t}| \geq s, \\
\int_{\mathbb{R}^{k}}\left(\mathcal{G}_{k}(\mathbf{x} ; r, s)-\mathcal{F}_{k}(\mathbf{x} ; r, s)\right) d \mathbf{x}=A_{k}(r, s),
\end{gathered}
$$

where $A_{k}(r, s)$ is defined in the statement of the theorem. From (1.3) we see that for $\mathbf{c} \in \mathbb{R}^{k}$,

$$
\Delta\left[\mathcal{P} ; B_{k}(r, \mathbf{c})\right]=\sum_{n=1}^{N} \sum_{\mathbf{m}} \chi_{r}\left(\mathbf{x}_{n}-\mathbf{c}+\mathbf{m}\right)-N \mu\left(B_{k}(r, \mathbf{0})\right) .
$$

Now suppose that for a given $r$ and $\mathbf{c}$ we have $\Delta\left[\mathcal{P} ; B_{k}(r, \mathbf{c})\right] \geq 0$. Then by (2.1), the Poisson summation formula and the triangle inequality we have

$$
\begin{aligned}
\Delta\left[\mathcal{P} ; B_{k}(r, \mathbf{c})\right] & \leq \sum_{n=1}^{N} \sum_{\mathbf{m}} \mathcal{G}_{k}\left(\mathbf{x}_{n}-\mathbf{c}+\mathbf{m} ; r, s\right)-N \mu(B(r, \mathbf{0})) \\
& =\sum_{n=1}^{N} \sum_{\mathbf{m}} \widehat{\mathcal{G}}_{k}(\mathbf{m} ; r, s) e\left(\mathbf{m} \cdot\left(\mathbf{x}_{n}-\mathbf{c}\right)\right)-N \widehat{\chi}_{r}(\mathbf{0})
\end{aligned}
$$




$$
\begin{aligned}
& =\sum_{|\mathbf{m}|<s} \widehat{\mathcal{G}}_{k}(\mathbf{m} ; r, s) e(-\mathbf{m} \cdot \mathbf{c}) \sum_{n=1}^{N} e\left(\mathbf{m} \cdot \mathbf{x}_{n}\right)-N \widehat{\chi}_{r}(\mathbf{0}) \\
& \leq N\left(\widehat{\mathcal{G}}_{k}(\mathbf{0} ; r, s)-\widehat{\chi}_{r}(\mathbf{0})\right)+\sum_{|\mathbf{m}|<s}^{\prime}\left|\widehat{\mathcal{G}}_{k}(\mathbf{m} ; r, s)\right|\left|\sum_{n=1}^{N} e\left(\mathbf{m} \cdot \mathbf{x}_{n}\right)\right| .
\end{aligned}
$$

From (2.1) and (2.3) we know that

$$
\widehat{\mathcal{G}}_{k}(\mathbf{0} ; r, s)-\widehat{\chi}_{r}(\mathbf{0})=\int_{\mathbb{R}^{k}}\left(\mathcal{G}_{k}(\mathbf{x} ; r, s)-\chi_{r}(\mathbf{x})\right) d \mathbf{x} \leq A_{k}(r, s) .
$$

A general expression for $\widehat{\mathcal{G}}_{k}(\mathbf{t} ; r, s)$ seems difficult to find. However, we can obtain an estimate that will do for our purposes. First note that

$$
\widehat{\chi}_{r}(\mathbf{t})=\int_{\mathbb{R}^{k}} \chi_{r}(\mathbf{x}) e(-\mathbf{t} \cdot \mathbf{x}) d \mathbf{x}=(r /|\mathbf{t}|)^{k / 2} J_{k / 2}(2 \pi r|\mathbf{t}|) .
$$

Applying the triangle inequality together with (2.5) and the above identity, we see that

$$
\begin{aligned}
& \left|\widehat{\mathcal{G}}_{k}(\mathbf{t} ; r, s)\right| \\
& \quad \leq\left|\int_{\mathbb{R}^{k}}\left(\mathcal{G}_{k}(\mathbf{x} ; r, s)-\chi_{r}(\mathbf{x})\right) e(-\mathbf{t} \cdot \mathbf{x}) d \mathbf{x}\right|+\left|\int_{\mathbb{R}^{k}} \chi_{r}(\mathbf{x}) e(-\mathbf{t} \cdot \mathbf{x}) d \mathbf{x}\right| \\
& \quad \leq\left(\widehat{\mathcal{G}}_{k}(\mathbf{0} ; r, s)-\widehat{\chi}_{r}(\mathbf{0})\right)+\left|\widehat{\chi}_{r}(\mathbf{t})\right| \\
& \quad \leq A_{k}(r, s)+(r /|\mathbf{t}|)^{k / 2}\left|J_{k / 2}(2 \pi r|\mathbf{t}|)\right| .
\end{aligned}
$$

Thus it follows from (2.4) and (2.5) that

$$
\leq N A_{k}(r, s)+\sum_{|\mathbf{m}|<s}^{\prime}\left\{A_{k}(r, s)+(r /|\mathbf{m}|)^{k / 2}\left|J_{k / 2}(2 \pi r|\mathbf{m}|)\right|\right\}\left|\sum_{n=1}^{N} e\left(\mathbf{m} \cdot \mathbf{x}_{n}\right)\right| .
$$

If it should happen that for a given $r$ and $\mathbf{c}$ we have $\Delta\left[\mathcal{P} ; B_{k}(r, \mathbf{c})\right]<0$, then following the preceding analysis using $\mathcal{F}_{k}(\mathbf{x} ; r, s)$ in place of $\mathcal{G}_{k}(\mathbf{x} ; r, s)$ yields inequality (2.6) with a minus sign attached to the left-hand term. Combining these bounds verifies (1.5) and completes the proof.

Proof of Theorem 2. We begin by using some estimates to simplify the bound given in Theorem 1. If $r s \geq 1$, then $A_{k}(r, s) \ll_{k} s^{-1} r^{k-1}$ (see [7], Theorem 1). Combining this with the bound $\left|J_{\nu}(x)\right| \leq 1$ for $\nu>0$ and $x>0$ reduces $(1.5)$ to

$$
D_{r}(\mathcal{P}) \ll_{k} N s^{-1} r^{k-1}+\sum_{|\mathbf{m}|<s}^{\prime}\left\{s^{-1} r^{k-1}+(r /|\mathbf{m}|)^{k / 2}\right\}\left|\sum_{n=1}^{N} e\left(\mathbf{m} \cdot \mathbf{x}_{n}\right)\right| .
$$


For a prime $p$, if $\mathbf{h}$ and $\mathbf{m}$ are lattice points, then

$$
\sum_{n=1}^{p} e((n / p) \mathbf{h} \cdot \mathbf{m})= \begin{cases}0 & \text { if } \mathbf{h} \cdot \mathbf{m} \neq \equiv(\bmod p), \\ p & \text { if } \mathbf{h} \cdot \mathbf{m} \equiv 0(\bmod p) .\end{cases}
$$

Therefore we have

$$
D_{r}\left(\mathcal{P}_{\mathbf{h}}\right) \ll_{k} p s^{-1} r^{k-1}+\sum_{\substack{|\mathbf{m}|<s \\ \mathbf{h} \cdot \mathbf{m} \equiv 0}}^{\prime}\left\{s^{-1} r^{k-1}+(r /|\mathbf{m}|)^{k / 2}\right\} p .
$$

The sum above is difficult to handle alone, but the problem simplifies if we average over all lattice points $\mathbf{h}$ such that $|\mathbf{h}| \leq p$. On doing so, the right side of (2.7) is equal to

$$
\begin{gathered}
p s^{-1} r^{k-1}+p\left(Z\left[\mathbb{Z}^{k} ; B_{k}(p, \mathbf{0})\right]\right)^{-1} \sum_{|\mathbf{h}| \leq p} \sum_{\substack{|\mathbf{m}|<s \\
\mathbf{h} \cdot \mathbf{m} \equiv 0}}^{\prime}\left\{s^{-1} r^{k-1}+(r /|\mathbf{m}|)^{k / 2}\right\} \\
=p s^{-1} r^{k-1}+p\left(Z\left[\mathbb{Z}^{k} ; B_{k}(p, \mathbf{0})\right]\right)^{-1} \sum_{|\mathbf{m}|<s}^{\prime}\left\{s^{-1} r^{k-1}+(r /|\mathbf{m}|)^{k / 2}\right\} \sum_{\substack{|\mathbf{h}| \leq p \\
\mathbf{h} \cdot \mathbf{m} \equiv 0}} 1 .
\end{gathered}
$$

For each $\mathbf{m}$ in the outer sum on the right side of (2.8), there is at least one nonzero component $m_{g}$. We fix such an $\mathbf{m}$, and consider the inner sum. For a given $\mathbf{h}$, once the components $h_{1}, \ldots, h_{g-1}, h_{g+1}, \ldots, h_{k}$ are set, there is only one choice for $h_{g}(\bmod p)$ for which $\mathbf{h} \cdot \mathbf{m} \equiv 0(\bmod p)$. Since $|\mathbf{h}| \leq p$, there are at most three possible choices for $h_{g}$. Furthermore, the other components $h_{1}, \ldots, h_{g-1}, h_{g+1}, \ldots, h_{k}$ must satisfy $\left|h_{j}\right| \leq p$ for each appropriate $j$. Thus for $\mathbf{m} \neq \mathbf{0}$,

$$
\sum_{\substack{|\mathbf{h}| \leq p \\ \mathbf{h} \cdot \mathbf{m} \equiv 0}} 1 \ll_{k} p^{k-1}
$$

We also note that for each $i \geq 2$, there exist constants $c_{4}(i)$ and $c_{5}(i)$ such that

$$
c_{4}(i) p^{i} \leq Z\left[\mathbb{Z}^{i} ; B_{i}(p, \mathbf{0})\right] \leq c_{5}(i) p^{i} .
$$

(See [6], Theorem 339, for a discussion of the case $i=2$.) As we are averaging, we see that (2.7)-(2.10) imply there exists a lattice point $\mathbf{h}$ with $|\mathbf{h}| \leq p$ such that

$$
D_{r}\left(\mathcal{P}_{\mathbf{h}}\right) \ll_{k} p s^{-1} r^{k-1}+\sum_{|\mathbf{m}|<s}^{\prime}\left\{s^{-1} r^{k-1}+(r /|\mathbf{m}|)^{k / 2}\right\} .
$$

Applying the inequality

$$
\sum_{|\mathbf{m}|<s}^{\prime}|\mathbf{m}|^{-k / 2} \ll_{k} s^{k / 2}
$$


and assuming that $r s \geq 1$ (required for our bound on $A_{k}(r, s)$ to be valid), we find that

$$
D_{r}\left(\mathcal{P}_{\mathbf{h}}\right) \ll_{k} p s^{-1} r^{k-1}+(s r)^{k-1} .
$$

The expression on the right above is minimized upon setting $s=p^{1 / k}$, which yields

$$
D_{r}\left(\mathcal{P}_{\mathbf{h}}\right) \ll_{k}\left(p r^{k}\right)^{(k-1) / k},
$$

and completes the proof.

\section{References}

[1] R. C. Baker, Diophantine Inequalities, Oxford University Press, New York, 1986.

[2] J. Beck and W. W. L. Chen, Irregularities of Distribution, Cambridge University Press, 1987.

[3] T. Cochrane, Trigonometric approximation and uniform distribution modulo 1, Proc. Amer. Math. Soc. 103 (1988), 695-702.

[4] P. Erdős and P. Turán, On a problem in the theory of uniform distribution, I, Indag. Math. 10 (1948), 370-378.

[5] P. J. Grabner, Erdős-Turán type discrepancy bounds, Monatsh. Math. 111 (1991), $127-135$

[6] G. H. Hardy and E. M. Wright, An Introduction to the Theory of Numbers, 5th ed., Oxford University Press, Oxford, 1979.

[7] J. J. Holt and J. D. Vaaler, The Beurling-Selberg extremal functions for a ball in Euclidean space, to appear.

[8] L. Kuipers and H. Niederreiter, Uniform Distribution of Sequences, Wiley, New York, 1974.

[9] W. M. Schmidt, Irregularities of distribution, IV, Invent. Math. 7 (1969), 55-82.

[10] E. M. Stein and G. Weiss, Introduction to Fourier Analysis on Euclidean Spaces, Princeton University Press, Princeton, N.J., 1971.

[11] P. Szüsz, Über ein Problem der Gleichverteilung, in: Comptes Rendus du Premier Congrès des Mathématiciens Hongrois, 1950, 461-472.

[12] J. D. Vaaler, Some extremal functions in Fourier analysis, Bull. Amer. Math. Soc. 12 (1985), 183-216.

[13] —, Refinements of the Erdős-Turán inequality, in: Number Theory with an Emphasis on the Markoff Spectrum, W. Moran and A. Pollington (eds.), Marcel Dekker, New York, 1993, 263-269.

DEPARTMENT OF MATHEMATICS

MICHIGAN TECHNOLOGICAL UNIVERSITY

HOUGHTON, MICHIGAN 49931

U.S.A. 